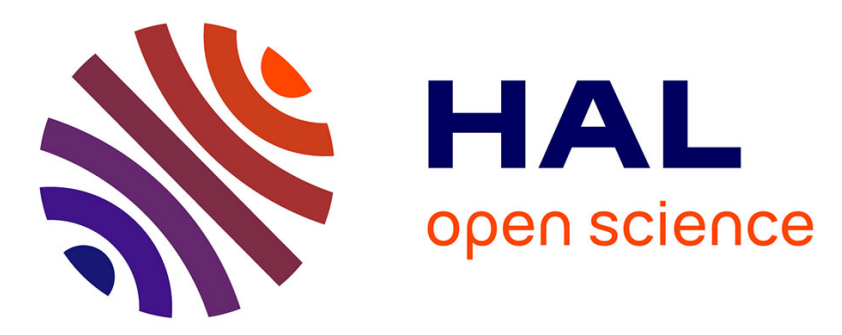

\title{
Automated Emergence of a Crisis Situation Model in Crisis Response Based on Tweets
}

Aurelie Montarnal, Shane Halse, Andrea Tapia, Sébastien Truptil, Frederick Benaben

\section{- To cite this version:}

Aurelie Montarnal, Shane Halse, Andrea Tapia, Sébastien Truptil, Frederick Benaben. Automated Emergence of a Crisis Situation Model in Crisis Response Based on Tweets. 18th Working Conference on Virtual Enterprises (PROVE), Sep 2017, Vicenza, Italy. pp.658-665, 10.1007/978-3-319-651514_58. hal-01674873

\section{HAL Id: hal-01674873 \\ https://hal.inria.fr/hal-01674873}

Submitted on 3 Jan 2018

HAL is a multi-disciplinary open access archive for the deposit and dissemination of scientific research documents, whether they are published or not. The documents may come from teaching and research institutions in France or abroad, or from public or private research centers.
L'archive ouverte pluridisciplinaire HAL, est destinée au dépôt et à la diffusion de documents scientifiques de niveau recherche, publiés ou non, émanant des établissements d'enseignement et de recherche français ou étrangers, des laboratoires publics ou privés.

\section{(c)(1)}

Distributed under a Creative Commons Attribution| 4.0 International License 


\title{
Automated Emergence of a Crisis Situation Model in Crisis Response based on Tweets
}

\author{
Aurélie Montarnal ${ }^{1}$, Shane Halse ${ }^{2}$, Andrea Tapia ${ }^{2}$, Sébastien Truptil ${ }^{1}$ \\ and Frédérick Bénaben ${ }^{1}$, \\ ${ }^{1}$ IMT Mines Albi, University of Toulouse, France \\ ${ }^{2}$ Penn State University, USA \\ \{aurelie.montarnal, sebastien.truptil, frederick.benaben\}@mines-albi.fr, \\ $\{$ seh297, atapia\}@ist.psu.edu
}

\begin{abstract}
During a crisis, being able to understand quickly the situation on-site is crucial for the responders to take relevant decisions together. Social media, in particular Twitter, have proved to be a means for rapidly getting information from the field. However, the deluge of data is heterogeneous in many ways (location, trust, content, vocabulary, etc), and getting a model of the crisis situation still requires laborious human actions. In addition, depending on which kind of information is mined from them, tweets have to be handle oneby-one (e.g. find victims), or as a whole - amount of tweets - (e.g. occurence of an event). This paper proposes a framework for automatically extracting, interpreting and aggregating streams of tweets to characterize crisis situations. It is based on a specific metamodel that determines the different concepts required to model a crisis situation.
\end{abstract}

Keywords: crisis situation modeling, decision support system, crisis management, cross-organizational collaboration.

\section{Introduction}

It is commonly accepted that crisis management can be divided into four phases: prevention, preparation, response and recovery [1]. While the prevention, preparation and recovery phases deal with avoiding or being more ready to crisis situations, the response phase has to deal with adaptation and improvisation in an emergency situation. In particular, the actors involved in the crisis response have to operate in a relevant and timely manner, which requires from decision-makers to make decisions in consequence. Charles et al. [2] emphasizes the challenge of having heterogeneous partners mounting a one-shot suitable Virtual Organization in time of crisis situations.

It has been observed that a limitation in this process is related to the difficulties to manage their coordination and collaboration [3]. About this, Wolbers and Boersma [4] argue that enhancing the responders' situational awareness indeed leverages the coordination of the actors, and this implies to be able to provide them with a common 
operational picture that includes the important concepts (i.e. elements on the field) that need to be taken into account.

In parallel, disaster management comes with an ever-increasing amount of generated and potentially accessible data. On any crisis site, heterogeneous and potentially undedicated data sources (sensors, opendata, witnesses and social networks, etc.) generate a deluge of data. As an example, Alexander [5] highlights that only 50 minutes after the 2012 earthquake in Lombardy, a clear and comprehensive picture of the situation was available mostly established from social media information.

This paper aims at exploring the automated exploitation of social media data (in particular tweets for their easy access thanks to the Twitter API), that have shown an invaluable potential but are so far humanly exploited in most of the cases, to convey a situation model of the crisis that will in turn be used as a basis to support the responders' collaborations.

Two main issues have to be tackled to implement such a system:

- How to aggregate tweets to detect "breaking news" within the massive flow of data?

- How to instantiate those data into instances of the situational model?

In our study, only the tweets gathered on specific geographical area (according to their geotagging metadata) are considered, as they can be assumed as the most informative in real-time.

The first part of the paper brings some visibility to the readers about which are the concepts mined to build a situational model, and then takes an example of flow of tweets during the specific crisis of the Boston Marathon in 2013 to highlight the challenges that need to be tackled. Then, a state of the art shows the already existing methodologies that have emerged these last years and their limitations for our specific use. Finally, a new proposition is explored and discussed.

\section{Background of the Research Works}

\subsection{Which Concepts to Mine to Build a Situational Model?}

According to Vieweg [6] situational awareness is defined as "understanding the big picture in time and safety critical situations. According to [7], situation awareness is performed through three levels: perception, comprehension and projection. These three terms are defined by as follows [8]: the perception refers to the ability of the system to get the "status, attributes and dynamics of relevant elements" in the environment; comprehension to provide a "synthesis" to support decision makers; projection to forecast near term future actions. While the first part can be provided by data sources (i.e. social media and in particular Twitter in our case), the second part required to be framed to know which kind of concepts have to be included in the synthesis. The third part will not be explored in this paper. We propose the synthesis to be a crisis situation model, just defined as proposed in the metamodel of Benaben 
et al. [9]. As a summary, two main packages are required to describe the crisis situation itself: (i) the context and (ii) the objectives packages. The context includes all elements of the environment, at stake, such as goods, people, natural sites, civilian society, within a specific territory, and also consequently inferred elements such as the corresponding dangers and intrinsic risks. In particular, in the objectives package, the event and emerging risks are the most interesting concepts to extract from datasets, since it can be used to infer the consequent facts.

\subsection{Problem Statement Illustrated through the 2013 Boston Marathon Example}

To better clarify the challenge to tackle to automatically define crisis situation models, from social media data, an example is given. A set of 5473 tweets on the 2013 Boston Marathon Bombing, geotagged in Boston area from 2013-04-15 20:49 CET (14:49 local time) to 2013-04-16 01:59 CET (19:59 local time), was manually classified along several classes partly related to the metamodel. Two bombs exploded at the marathon finish line at 14:50 local time; after a short while a fire is declared at the close JFK Library; several minutes later, fake rumors of other devices were spread from several places in the city. A short observation quickly led to several evidences. The diagram in Fig. 2 below shows the evolution of the number of tweets related to specific classes, cumulated on 10 minutes' windows.

We observed that: (i) such classes as Event, produce expected spikes, but those can occur after the event in question (see frequent increasing slope after 22:00 CET), which can eventually produce a 'noise'; (ii) sometimes isolated tweets (e.g. about victims, or even rumors) conveyed as much information as sets of tweets (e.g. occurrence of an event).

In general, considering the two following statements:

-Depending on the type of concept mined, either isolated tweets or the important amount of similar tweets triggers the instantiation on the model.

-An important amount of tweets related to a class becomes eventually a noise which prevents to detect new information.

the challenge that these works aims to tackle is: How to aggregate tweets, so that isolated or packs of tweets can be considered equally meaningful, and how to detect in an amount of similar tweets, if the information provided is new, or just an 'echo' throughout the crisis? 


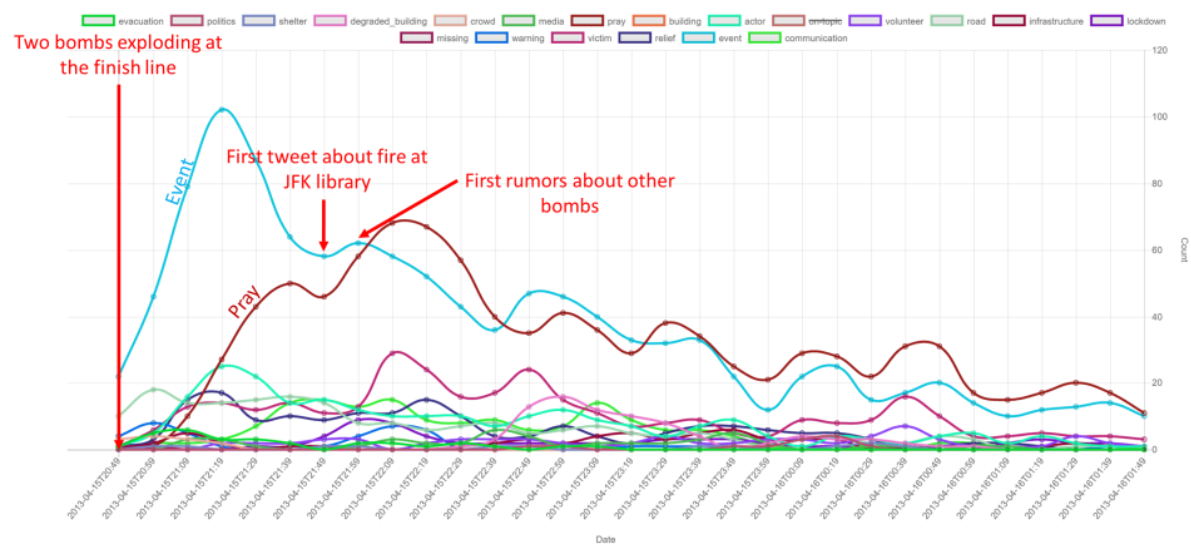

Fig. 1. Number of tweets for each studied class, along the crisis response.

\section{State of the Art}

When exploring a way to use Big-Data as a means to support the inter-organizational collaborations that articulate the responders' actions and decisions, [10] highlights that this is a challenge on both (i) a social layer with the necessity to support the interorganizational collaborations (among the crisis' responders) at a macro-level and, in the same time, (ii) a technological layer with the lack of methodology to deal with unknown sources of data that needs to be "understood" to be exploited by decisionmakers. Benaben et al. [10] especially summarize that, in disaster management, being able to use and "understand" data is required to support the coordination issues. Hence, focusing on the use of social media data, the following presents a specific study on existing methodologies to exploit and interpret this kind of data in such a context.

Yin et et al. [7] have developed a tool to mine and extract data from micro blogs related to the crisis in a near-real-time manner. This tool allows users, both in a crisis decision making or general public role, to visualize information about the incident and the potential impact it may have. The tool is based on burst detection, numerous messages with similar wording or from the same area with an automated data collection. Sakaki et al. use Twitter data flows to detect earthquake in real-time [11]. They built a reporting tool that embeds two parts: the detection of tweets that are about earthquakes and a service dedicated to location detection. [12] provide a fourstep approach to take advantage of tweets in crisis situations: (i) burst detection, (ii) text classification to find tweets about infrastructures status, (iii) online clustering and (iv) geotagging of the tweets.

Overall, these solutions are rather about providing decision-makers with visualization tools. As such, even if it is of help for the users, the resulting outputs remain at a data level of abstraction, i.e. the users still need to put them into a context by themselves if they want to obtain usable information for their decision-making 
process.

Many works have based their approach on classification problems, i.e. labelling each tweet with specific class(es), that have been predefined. Among them, [13] and [14] propose tweets datasets with many crises cases. In the first case, the tweets have been classified informative or not thanks to a crowdsourcing service. In the second case, as a first step, tweets have been classified between on-topic and off-topic thanks to crowdsourcing, and the authors have use this training dataset to extract specific terms related to crisis (app. 380 terms). In line, [15] built a lexicon EMTerms 1.0 of more than 7200 terms directly related to 23 specific crisis categories. Both lexicons are available online ${ }^{1}$. Using lexicons with keywords occurrence can show some good enough results, providing that the lexicons were built on suitable use cases (i.e. if they are built on hurricane and earthquake cases, there are good chances that the results will be bad in the case of a terror attack), however, extending lexicons with new cases is time-consuming and results are not always guaranteed, because it is rather hard to detect the terms that could make the lexicons too specific.

In Natural Language Processing (NLP), many methods can be used to classify corpus of documents (in our case a set of tweets, each one belonging to a specific class). Among them, a Convolutional Neural Network model, as presented in [16] allows to adapt an Artificial Neural Network model for analyzing n-grams. [17] provide comparative results of tweets classification over several methods: Support Vector Machines, Artificial Neural Networks (ANN), Convolutional Neural Networks $(\mathrm{CNN})$ through unigrams, unigrams+bigrams and unigrams+bigrams+trigrams. As a result, they show that in their two-class (on-topic or off-topic) classification case, CNNs are the most suitable.

Finally, this state of the art shows that, in order to "go up" in abstraction (i.e. instantiate the concepts into the model), the current systems miss to take into account the interactions among the tweets, which could be enabled thanks to a deeper semantic analysis.

\section{Solution Proposal}

\section{1 "Concept-similarity" Graph Emergence}

Our proposal is to take advantage both of the classification methods that have shown decent results in the literature, but are too generic to lead to any instantiation, and semantics that allows to get a deeper meaning out of tweet data.

Our framework includes an analysis of the tweets decomposed into three dimensions:

-A time dimension: a sliding time window should be set as a parameter, during which the analysis is executed (e.g. all tweets from t-30 minutes to $t$ are analyzed).

1 http://www.crisislex.org 
This dimension ensures a memory to the system, and, in the same time, allows it to "forget" information to avoid "echo" or "amalgam" effects resulting from a massive amount of outdated information.

-A class dimension: this dimension includes all concepts (classes of the metamodel) that should be extracted from the tweets. These classes are used as sets that can potentially overlap, and on which the set theory principles can be applied. For each tweet retrieved, it can either belong to none of the classes (i.e. non-informative tweet), or to 1 to several classes.

-A semantic dimension: the goal of this dimension is, considering the time window and the later classification of the tweets, to define semantic similarities between tweets, to aggregate them.

As a result, for a sliding time window, at all time, a "concept-similarity" graph can be established as illustrated in Fig. 2.

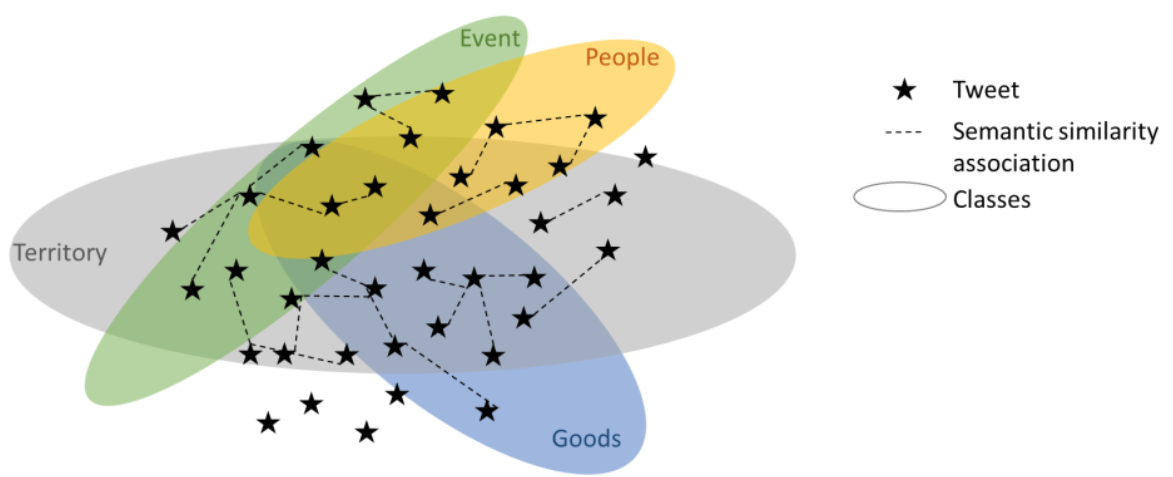

Fig. 2. "Concept-similarity" graph: semantic aggregation of classified tweets.

\subsection{Exploitation of the "Concept-similarity" Graph}

Based on this type of graph, rules can be established to determine if a set of similar tweets ( 1 or more) should trigger a new instantiation on the crisis situation model.

One example of such rule could be (the function Near(Tweet1, Tweet2) relates to a near semantic association between Tweet1 and Tweet2):

Let $T$ be the monitored Territory,

Let $E$ be the set of all Events,

Let $T w_{1}$ be a "new" tweet,

Let $T w_{2}$ be a tweet involved in the instantiation of an event $E_{1} \subset E$ (i.e. $T w_{2} \in E_{1}$ )

If $\left(\left(T w_{1}, T w_{2}\right) \in T \cap E\right) A N D\left(\operatorname{Near}\left(T w_{1}, T w_{2}\right)\right)$ Then,

Aggregate $T w_{1}$ and $T w_{2}$ together (i.e. $T w_{1} \in E_{1}$, no new instantiation)

The overall expected architecture to be implemented can be seen in Fig. 3: two services are expected to work in parallel to classify the tweets and, in the same time, establish semantic relationships among them. A rules engine then run predefined rules to trigger or not instantiation into the crisis situation model. The graph obtained by the 
two first services is updated all along the crisis, according to the time window defined as a parameter.

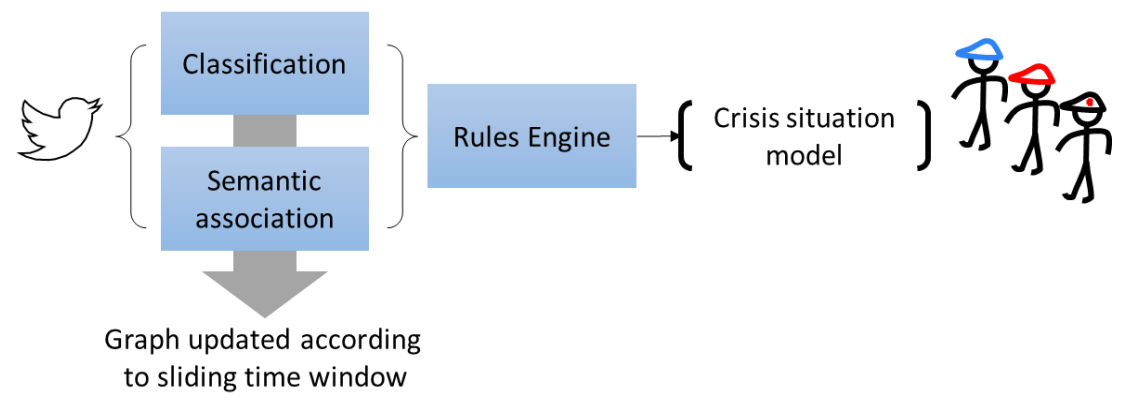

Fig. 3. Functional architecture proposed.

\section{Conclusion and Perspectives}

Getting a crisis situation model from data flows like tweets is a burning topic and a challenge, considering both the invaluable information that could be extracted and the laborious human effort it still requires. While many works rather focus on providing crisis management decision makers with visualizations, based on classification approaches, discovering the concepts and instantiate them into a crisis situation model is still up to them. Automating this instantiation (i.e. extracting unique and informative instances of the crisis situation model) goes through the aggregation of tweets. In parallel, having to mount on-the-fly collaborations to respond to a crisis is a tough task, hence, such virtual organizations would deeply beneficiate from this automated instantiation of a crisis situation model leveraging both the efficiency and the speed of the collaborative response.

To tackle this challenge, this paper aims to explore a new approach that embeds both the strength of classification methodologies and semantics, to exploit and interpret geotagged tweets on a particular monitored area. A system in three functional parts is proposed as follows: (i) a classification service of tweets along predefined classes used to model the situation, (ii) a service dedicated to the emergence of semantic associations among the tweets to aggregate them and (iii) a rules engine to automatically exploit the graph obtained in (i) and (ii) and trigger the instantiations.

The proposed framework will be used to implement such system and assess it through different real use-cases extracted from past crises. In further step, this system could be improved by adding a correlation service, to compare extraction from social media data and sensors data: this could improve the reliability and the trust of the final crisis situation model. 


\section{References}

1. Altay, N., \& Green, W. G.: OR/MS research in disaster operations management. European journal of operational research, 175(1), 475-493 (2006)

2. Charles, A., Lauras, M., Barthe, A. M., \& Bénaben, F.: Gathering, Structuring and Modeling Business Process Knowledge of the Response to a Nuclear Crisis: Towards a Simulation Platform for Better Coordination. In Working Conference on Virtual Enterprises (pp. 486-493). Springer Berlin Heidelberg (2011)

3. Benaben, F., Truptil, S., Lauras, M., \& Salatge, N.: Management of collaborative behavior through a service-oriented mediation system: the case of crisis management. In 2015 IEEE International Conference on Services Computing (SCC), pp. 554-561. IEEE (2015)

4. Wolbers, J., \& Boersma, K.: The common operational picture as collective sensemaking. Journal of Contingencies and Crisis Management, 21(4), 186-199 (2013)

5. Alexander, D. E.: Social media in disaster risk reduction and crisis management. Science and Engineering Ethics, 20(3), 717-733 (2014)

6. Vieweg, S. E.: Situational awareness in mass emergency: A behavioral and linguistic analysis of microblogged communications (2012)

7. Yin, J., Karimi, S., Lampert, A., Cameron, M., Robinson, B., \& Power, R.: Using social media to enhance emergency situation awareness. In Twenty-Fourth International Joint Conference on Artificial Intelligence. (2015)

8. Endsley, M. R.: Toward a theory of situation awareness in dynamic systems. Human Factors: The Journal of the Human Factors and Ergonomics Society, 37(1), 32-64 (1995)

9. Benaben, F., Montarnal, A., Truptil, S., Lauras, M., Fertier, A., Salatge, N., \& Rebiere, S.: A Conceptual Framework and a Suite of Tools to Support Crisis Management. In Proceedings of the 50th Hawaii International Conference on System Sciences (2017)

10. Benaben, F., Montarnal, A., Fertier, A., \& Truptil, S.: Big-Data and the Question of Horizontal and Vertical Intelligence: A Discussion on Disaster Management. In Working Conference on Virtual Enterprises (pp. 156-162). Springer International Publishing (2016)

11. Sakaki, T., Okazaki, M., \& Matsuo, Y.: Earthquake shakes Twitter users: real-time event detection by social sensors. In Proceedings of the 19th international conference on World wide web (pp. 851-860). ACM. (2010)

12. Cameron, M. A., Power, R., Robinson, B., \& Yin, J.: Emergency situation awareness from twitter for crisis management. In Proceedings of the 21 st International Conference on World Wide Web (pp. 695-698). ACM (2012)

13. Olteanu, A., Vieweg, S., \& Castillo, C.: What to expect when the unexpected happens: Social media communications across crises. In Proceedings of the 18th ACM Conference on Computer Supported Cooperative Work \& Social Computing (pp. 994-1009). ACM (2015)

14. Olteanu, A., Castillo, C., Diaz, F., \& Vieweg, S.: CrisisLex: A Lexicon for Collecting and Filtering Microblogged Communications in Crises. In ICWSM (2014)

15. Temnikova, I., Castillo, C., \& Vieweg, S.: EMTerms 1.0: a terminological resource for crisis tweets. In ISCRAM 2015 proceedings of the 12th international conference on information systems for crisis response and management (2015)

16. Johnson, R., \& Zhang, T.: Effective use of word order for text categorization with convolutional neural networks. arXiv preprint arXiv:1412.1058 (2014)

17. Caragea, C., Silvescu, A., \& Tapia, A. H.: Identifying informative messages in disaster events using convolutional neural networks. In International Conference on Information Systems for Crisis Response and Management (2016) 